\title{
Data Sharing Mandates, Developmental Science, and Responsibly Supporting Authors
}

\author{
Roger J. R. Levesque ${ }^{1}$
}

Received: 21 August 2017 / Accepted: 28 August 2017 / Published online: 13 September 2017

(C) Springer Science+Business Media, LLC 2017

\begin{abstract}
Data sharing has come of age. Long expected as a professional courtesy but rarely honored, data sharing is now highlighted in codes of ethics, supported by research communities, required by leading funding organizations, and variously encouraged and mandated by journals and even publishers. These developments reveal how sharing generates many benefits, all of which go to the integrity of the scientific process. Yet, sharing remains a complex phenomenon. This Editorial explains the journal's response to the publisher's mandate to establish an appropriate data sharing policy for the Journal of Youth and Adolescence. It describes the need to balance the benefits of sharing with its costs for authors publishing in multidisciplinary, developmental science journals like this one. For this journal and at this time, that balance leads us to err on the side of caution, which means supporting those who created their data and not coercing public sharing as a condition for publishing. This approach recognizes authors' reliance on a wide variety of data, the needs of differentially situated authors, the requirements of robust peer review, and the potential harms that can come from editors' unilateral sharing mandates.
\end{abstract}

Keywords Data Sharing - Replication - Authors' rights • Editorial Guidelines

Roger J. R. Levesque

rlevesqu@indiana.edu

1 Indiana University, 302 Sycamore Hall, Bloomington, IN, USA

\section{Developments in the Journal's Data Sharing Policy}

In 2016, the publisher of the Journal of Youth and Adolescence required its journals to institute data sharing policies. These policies encourage or mandate authors to share their original data with the research community, including journal editors, reviewers, and researchers seeking to reproduce analyses or otherwise use the data for novel research. The publisher gave journal editors flexibility in the policy that they would adopt, a flexibility that ranged from merely encouraging to mandating data sharing for public use (see Table 1).

In responding to the publisher's request, this journal had adopted a minimalist approach, what the publisher deems Type 1. That approach appeared reasonable given that a look at journals that cite to our journal and the journals that our journal cites to revealed that the few that did have statements encouraged (did not mandate) sharing. As a result, authors currently indicate the following when submitting their manuscripts:

- My manuscript has associated data in a data repository.

- My manuscript has no associated data or the data will not be deposited.

- My manuscript has data included as electronic supplementary material.

The current approach means that the editor and publisher's staff readily see the authors' declarations. Reviewers and others who seek to understand or use the study's findings do not have ready access to the statements, let alone the associated data.

We now question the practice of not making data sharing declarations public. There appears to be no compelling reason for not publishing declarations in the articles themselves, particularly since the declarations being 


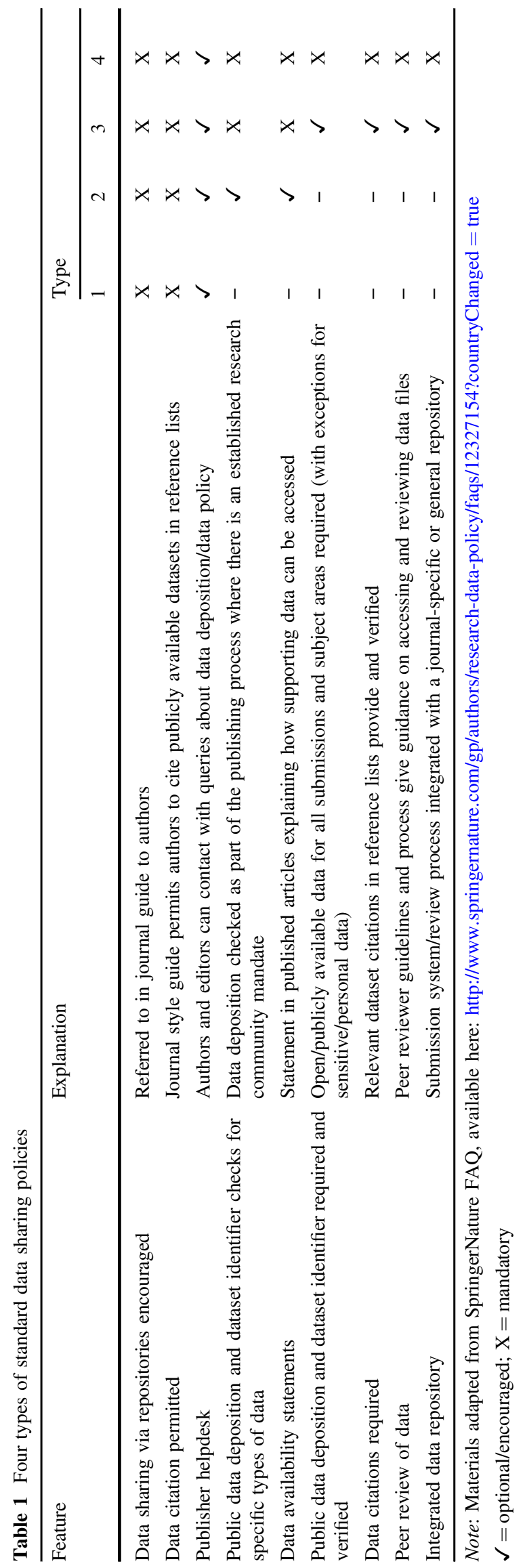

made to the publisher are the same that would be made public. Making those statements public also is not more problematic given that the journal only encourages post publication data sharing. This is what Type 2 seeks to do.

Adopting Type 2 means that authors submitting to the journal will soon find that we require more than simply notifying the editor about the data being used and how it can be shared once the manuscript is published. Authors will need to make a public statement about their data's availability and how it can be obtained if it is going to be deposited or otherwise shared.

The major shift from Type 1 to Type 2 is that authors who agree to share their original data now must also reveal how others can access it. Following Type 2 to its logical conclusion means that authors can note several possible Data Sharing Declarations in their acknowledgments:

- This manuscript's data will not be deposited.

- Data sharing is not applicable to this article as no datasets were generated or analyzed during the current study.

- All data generated or analyzed during this study are included in this published article (and its supplementary information files).

- The datasets generated and/or analyzed during the current study are not publicly available but are available from the corresponding author on reasonable request.

- The datasets generated and/or analyzed during the current study are available in the [NAME] repository, [PERSISTENT WEB LINK TO DATASETS].

- The data that support the findings of this study are available from [THIRD PARTY NAME] but restrictions apply to the availability of these data, which were used under license for the current study, and so are not publicly available. However, data are available from the authors upon reasonable request and with permission of [THIRD PARTY NAME].

Notably, the last two declarations provide only a starting point for discussion. Those seeking to use the original data will need to know what will be available, such as which data, data dictionaries, statistical analysis plans, study protocols, dates of data availability, and criteria for access. Much is needed for meaningful or correct reanalysis, a point that figured prominently in the journal's position on data sharing.

\section{The Journal's Position on Data Sharing}

A reading of editorials explaining data sharing policies and a look at the policies themselves clearly leaves the 
impression that the goal is to pressure authors toward sharing data. For example, some journals encourage and even mandate sharing of some data (see Lindsay 2017). Others pressure authors to deposit their data by requiring them to explicitly state why they are not sharing and requiring authors to have compelling reasons for not sharing (see Barbui et al. 2016). In some disciplines, editors have grouped together and agreed to move toward mandated data sharing for some types of data (see Taichman et al. 2017). These developments lead editors in some fields, such as medicine, to view the practice of data sharing, in one form or another, as inevitable (Merson et al. 2016; Bauchner et al. 2016). Together, these developments leave no doubt that editors taking positions on data sharing are moving toward Type 4 described in Table 1.

This journal takes a different position. We do not adopt what appears to be the emerging trend, including what appears to be assumptions about "encouraging" data sharing. Instead, we take on the burden of supporting authors. The major form of support is to help authors alert readers where data can be located if authors wish to share their data. For authors who need assistance in depositing their data, the publisher offers support, including pointing authors to curation services. The publisher also has a help desk to assist researchers who are creating data to share. In our view, that support is what the field, and journal, needs. Authors should not be compelled to share their data publicly, nor should they feel compelled. This includes not being required to state why they choose not to share their data. They should be supported to make decisions they deem appropriate.

We view the journal as taking on the burden of helping authors make what they view as the responsible decision regarding the sharing of their data. Placing the responsibility on the authors, rather than on the journal to nudge or mandate sharing, does not mean that authors are free to ignore calls to share their data. It is notable that, regardless of whether they deposit their data or indicate where it can be found, authors still commit to making the data available upon a reasonable request. Such commitments have long been accepted professional custom and are stated in a variety of professional codes of ethics, most notably for our journal, the code adopted by the American Psychological Association (2017, Standard 8.14).

\section{Rationales for not Compelling Authors to Share their Data}

Two major concerns foster recent developments in sharing data. First, researchers tend to be unwilling to share, as even authors who publish in journals that mandate sharing pervasively do not share (Savage and Vickers 2009; Fecher et al. 2015). To complicate matters, those who are willing also tend to be ineffective sharers (see Tenopir et al. 2011). Second, the integrity of the scientific enterprise relies on transparency, accountability and replicability that can be more readily had with access to primary data (see Barbui et al. 2016). Easy access to data and materials facilitates scientific developments as it allows for better evaluations of substantive claims and produces more informed research. In addressing these two concerns, the general needs of the scientific enterprise have started to supersede those of individual authors.

Concerns about limited data sharing have merit, but they do not necessarily counsel opening data for public consumption. That is why editors' move toward coercing data sharing, although well-intentioned, appears to be heading in the wrong direction. For those of us, like this editor, who study the failures of policies and legal systems, we are not surprised to find that authors are notoriously incompetent sharers of their data even though they commit to doing some sharing when they publish their work. Many factors inhibit sharing, such as authors' concerns about originality, the protection of participants, intellectual property, data collection incentives, curation, translations and privacy (see, e.g., Gewin 2016; Voytek 2016). However, likely the most compelling reasons for not making data available relate to authors' costs associated with investing resources into making data available and taking risks when the data are available. These are not unimportant considerations.

While reasons for not sharing have been dismissed as unfounded (see Smith and Roberts 2016), coercive sharing mandates do not necessarily address authors' concerns. Costs and risks do not disappear just because mandates require compliance. Yet, that is precisely what several policies appear to assume. As an example, it has been suggested that codes of ethics protect authors from the harms that could come from shared research. In announcing its recent move toward sharing data, Psychological Science pointed to the code of ethics as the major source of protection against the problematic use of shared data (Lindsay 2017). Yet, given that the failure to follow codes of ethics about data sharing led to journal policies encouraging the deposition of data, it is somewhat surprising that codes of ethics are referenced as the obvious source of protection against the problematic use of data provided for public consumption. It would seem reasonable to question how much "teeth" a code has in shaping protective behavior when it failed to shape benevolent sharing. From this editor's perspective, harms arising from shared data are not easily remediable and preventable, which raises the duty of 
journal editors to protect authors' interests rather than place them at risk.

There may be many compelling reasons for not mandating data sharing in our area of research, ${ }^{1}$ but arguably the most compelling reason for not mandating is that the field (particularly its journal editors who would impose mandates) has not developed methods to shield authors from the problematic use of their data. This is particularly important given that researchers are the ones burdened when others wrongly use their original data. For some researchers who collect their own data, the benefits that can come from broader data sharing may not justify the potential harms.

Anecdotal evidence from editorial board members points to potential harms associated with the variety of ways that authors share their findings. Current practices already place authors at risk. For example, a board member recently noted surprise when asked to review a manuscript based on an analysis that they had sent out for review to another journal; another expressed concern because they once shared unpublished data that others unexpectedly used as a foundation for a publication. Board members also have noted how problematic it is to share data when they seek to write multiple articles or are conducting longitudinal studies. Given the premium placed on publishing original work, the concern that others can publish more quickly or in stronger outlets is a very real one. And, let us not forget that the vast majority of researchers have spoken loudly by their actions: they tend not to share even when journals mandate sharing.

More forceful approaches to encouraging sharing could have its place in developing the field. As medical journals have shown, researchers and institutions have changed how they think about data collection and even the nature of data (see Barbui et al. 2016). Researchers now increasingly recognize the benefits of considering data sharing from the

\footnotetext{
${ }^{1}$ Many factors counsel against compelling authors to share their data and raise the duty to protect authors' interests. As of now, our field has not matured in ways similar to others that mandate sharing, such as fields of study that require very costly data collection or have exceedingly rare data that compel sharing. By and large, the studies published in our journal are not the type that participants would suffer if their data were not shared; and there also tends not to be public emergencies that mandate sharing and no excessive innovations that would limit other similarly situated researchers from obtaining their own data. In addition, some types of data may not be readily shared. While some sources of funding require researchers to plan for data sharing, some authors may not have access to such support. Basic fairness issues also ask whether it is fair to require some authors to share when others are not required to do so. Those same fairness concerns are grounds to question whether it is responsible to require some authors to bear the cost of preparing data for sharing when they lack needed support. Our field remains diverse in its data sources, a diversity that overly restrictive policies run the risk of stifling.
}

beginning of their projects, including when they obtain consent from participants and ensure confidentiality when data are shared (see Sarpatwari et al. 2014; El Emam et al. 2015), and they detail publication plans to make the best use of databases (Drazen 2014). And medical journal editors have taken steps to make data sharing the norm for some types of studies, as even they recently recognized that mandated sharing can be problematic and flexibility is needed (see Taichman et al. 2017). These developments certainly move toward more informed and efficient data sharing, but until efforts are made to better protect those who would share data, it is ill-advised to move hastily toward policies limiting publication to authors who share.

It is true that bad cases make bad law, that fear of problematic cases can result in problematic policies. Fears of system-gaming perhaps could be dismissed as reactionary. Concerns about "research parasites" have been raised and properly responded to as they run the risk of ignoring the legitimate use of available data and the benefits that come from replication as well as novel inquiry using existing data (see Emmert-Streib et al. 2016). Those points are well taken and highlight the need to not lump the legitimate use of deposited data sets with those that are obtained and used illegitimately.

However, those points are not the grounds on which we disagree about mandated data sharing. In fact, this journal has a long track record of supporting manuscripts that rely on large data sets collected and curated by others. In addition, this journal led to the development of another, Adolescent Research Review, dedicated to reviewing existing research (see Levesque 2016a) and encourages the field to better evaluate itself Levesque (2016b). And, this journal has a history of supporting emerging scholars who frequently rely on their mentors' data or large, curated data sets, as illustrated by nominees and recipients of this journal's annual emerging scholar awards (see, e.g., Levesque 2016c, 2015).

When it comes to authors making their data publicly available or when otherwise sharing their data with colleagues, this journal seeks to follow the simple rule that those who would take the most risk should control the decisions relating to the risk. The well-established rule for those of us in service professions, which undoubtedly includes editorial work, is that we first do no harm. This means that we must respect authors' decisions when they decline to make their data publicly available.

It also is true that requiring public statements about authors' data sharing decisions can be a source of pressure for authors who decline to make their data publicly available. For some authors, mandated public statements under the threat of editors' rejection may be considerably coercive. Journals routinely pressure authors (and authors 
may well not even realize the pressures that they are under). ${ }^{2}$ Notably, for example, this journal was the first of its type to "pressure" authors to make public statements about authors' relative contributions (see Levesque 2012). The journal also stands alone with the long list of acknowledgments it asks authors to publish, such as acknowledgments relating to funding, conflicts of interest, informed consent, and so forth. The inability to provide some of these statements has led to rejecting manuscripts. These types of pressure may be unique to the journal, but they are not cause for concern. All journals routinely prod, nudge and require authors to accommodate their own editorial demands.

Examples from the journal's past efforts indicate that the type of pressure that should concern us is undue pressure. We view the pressure to make public statements as part of the broader, typical pressure that researchers face when they need to be transparent. The peer review process has long been a check on problematic science. It certainly has its problems and is open to abuse, but authors who wish to publish must be willing to ensure that reviewers feel comfortable with the quality of their data. At least for this journal, reviewers routinely identify concerns about data and analyses, and those are the primary grounds for negative editorial decisions when authors are unable to address them reasonably. When reviewers request different analyses, authors frequently include them in the revised manuscripts as part of other sensitivity analyses. In addition, the journal currently has no space limitations once manuscripts have survived external peer review, which means that authors make considerable amounts of data public. The point is that the manuscripts we publish necessarily present extensive data and analyses for readers to evaluate their legitimacy. And, as noted above, authors are required to make many public acknowledgments to help readers discern potential biases and limitations. These considerations and processes may not be the best way for the system to correct and police itself, and it may be problematic for some authors, but it currently provides an appropriate balance between the need to produce robust, trustworthy science and the need to protect the interests of authors.

\footnotetext{
$\overline{2}$ Editors evaluate manuscripts in light of several considerations unknown to authors. Editors must consider not only the quality of submitted manuscripts but also the capabilities of their review process, impressions of the scholarly field's needs, subjective evaluations of the public's interest in the science, future and past manuscripts on specific topics or using specific analyses, and parameters expressed by the publisher. Publishers likely have more influence than most authors realize. Fortunately for this journal, however, the publisher's staff has been exceedingly supportive and has been at the forefront of advancing how our field publishes and what it publishes.
}

\section{Conclusion}

As a multidisciplinary, developmental science research outlet, this journal must recognize multiple interests at stake in calls for sharing data. Despite the benefits of sharing, it is important to avoid coercive mandates that jeopardize researchers. We are encouraged by the publisher's support of data sharing and developments that shape the accumulation of data. Some fields may legitimately require sharing; but ours has a long way to go before it can legitimately do so. Reasonable people may well disagree with our position. Ours rests on the accumulated knowledge of several colleagues and their sense of our fields of study. It also rests on what we actually study: social change, transitions, harms, and positive development as well as how best to protect and support responsible actions. Increasingly forceful calls for sharing, regardless of their apparent legitimacy, make it all the more important to not ignore our field's insights about how to foster and support people's responsible decisions and actions. Authors currently are the best situated to make data sharing decisions.

Acknowledgements This editorial benefitted from conversations with many colleagues associated with the journal, particularly the publisher's staff and Jonathan R. Brauer, who helped work through issues but are not responsible for this manuscript's conclusions and the journal's editorial positions.

\section{Compliance with Ethical Standards}

Conflict of Interest The author reports that he is the Editor of the Journal of Youth and Adolescence.

\section{References}

American Psychological Association. (2017). Ethical principles of psychologists and code of conduct. http://www.apa.org/ethics/ code/.

Barbui, C., Gureje, O., Puschner, B., Patten, S., \& Thornicroft, G. (2016). Implementing a data sharing culture. Epidemiology and Psychiatric Sciences, 25, 289-290.

Bauchner, H., Golub, R. M., \& Fontanarosa, P. B. (2016). Data sharing: an ethical and scientific imperative. JAMA, 315(12), 1238-1240.

Drazen, J. M. (2014). Open data. New England Journal of Medicine, $370,662-662$.

El Emam, K., Rodgers, S., \& Malin, B. (2015). Anonymising and sharing individual patient data. BMJ, 350, h1139.

Emmert-Streib, F., Dehmer, M., \& Yli-Harja, O. (2016). Against dataism and for data sharing of big biomedical and clinical data with research parasites. Frontiers in Genetics, 7, 154.

Fecher, B., Friesike, S., \& Hebing, M. (2015). What drives academic data sharing? PloS One, 10(2), e0118053.

Gewin, V. (2016). Data sharing: an open mind on open data. Nature, $529,117-119$.

Levesque, R. J. R. (2012). Authorships, collaborations, and acknowledgments: On the increasing responsibility to report 
relative contributions. Journal of Youth and Adolescence, 41(12), 1685-1687.

Levesque, R. J. R. (2015). Emerging scholar best article award, 2015. Journal of Youth and Adolescence, 44, 2395-2396.

Levesque, R. J. R. (2016a). The editor's inaugural introduction. Adolescent Research Review, 1, 1-2.

Levesque, R. J. R. (2016b). Reviews in research on adolescence: Genres, trends, and challenges. Adolescent Research Review, 1, 3-13.

Levesque, R. J. R. (2016c). Emerging scholar best article award, 2016. Journal of Youth and Adolescence, 45, 2497-2498.

Lindsay, D. S. (2017). Sharing data and materials in psychological science. Psychological Science, 2017, 1-4

Merson, L., Gaye, O., \& Guerin, P. J. (2016). Avoiding data dumpsters - toward equitable and useful data sharing. New England Journal of Medicine, 374(25), 2414-2415.

Sarpatwari, A., Kesselheim, A. S., Malin, B. A., Gagne, J. J., \& Schneeweiss, S. (2014). Ensuring patient privacy in data sharing for postapproval research. New England Journal of Medicine, $371,1644-1649$

Savage, C. J., \& Vickers, A. J. (2009). Empirical study of data sharing by authors publishing in PLoS journals. PloS One, 4(9), e7078.
Smith, R., \& Roberts, I. (2016). Time for sharing data to become routine: the seven excuses for not doing so are all invalid. F1000Research, 5, 781 pmid:27347380.

Tenopir, C., Allard, S., Douglass, K., Aydinoglu, A. U., Wu, L., Read, E., \& Frame, M. (2011). Data sharing by scientists: Practices and perceptions. PloS One, 6(6), e21101.

Taichman, D. B., Sahni, P., Pinborg, A., Peiperl, L., Laine, C., James, A., \& Frizelle, F. A. (2017). Data sharing statements for clinical trials-A requirement of the international committee of medical journal editors. New England Journal of Medicine, 376, 2277-2279.

Voytek, B. (2016). The virtuous cycle of a data ecosystem. PLoS Computational Biology, 12(8), e1005037.

Roger J. R. Levesque is Professor of Criminal Justice and (Affiliate) Law, Indiana University. He serves as Editor-in-Chief of the Journal of Youth and Adolescence and the Adolescent Research Review. He also is editor of the Advancing Responsible Adolescent Development book series. 